\title{
Selection of Non-Magnetic Fraction from the Slime Wastes of Metal Production in the Electromagnetic Field
}

\author{
Yuri Vernigorov ${ }^{1}$, Valery Lebedev ${ }^{1}$, and Lidianna Chunakhova $^{1}$ \\ ${ }^{1}$ Don State Technical University, 344000, Russia, Rostov-on-Don, DSTU, 1 Gagarin Sq.
}

\begin{abstract}
It is proved that the use of a variable inhomogeneous magnetic field, created by the superposition of constant and variable, strongly inhomogeneous fields, is considered to be very promising for the separation of non-magnetic fraction from powders of magnetic materials. This article introduces the effective technology of separation of nonmagnetic fraction from the sludge wastes of metal production in the electromagnetic field, which allows to destroy the aggregates, to separate the abrasive and to get products of high purity. A complex on processing of grinding sludge includes the following blocks: the loading block with dispenser; block of preliminary squeezing and washing of DRC; the block of drying; the block of a refinement; the block of division of sludge fractions. Experimental approbation of the proposed technological scheme of sludge separation, which had been carried out on iron ore concentrate of class 63 microns with silica content of $4.57 \%$ of the mass, showed that with the field parameters close to the calculated, the magnetic vibrating layer of iron powder PZh-4M has the maximum development; increasing the separation time leads to more efficient separation in the interval of field induction gradient from $550 \mathrm{mT} / \mathrm{m}$ to $610 \mathrm{mT} / \mathrm{m}$.
\end{abstract}

\section{Introduction}

At the enterprises of mechanical engineering and metal industry which carry out the processing of metals thousands of tons of metal-containing slimes are monthly formed. Grinding slime which represents a mixture of small metal shavings, abrasive, technical oils, drawing lubricant-coolant (DRC) and other components is especially complicated by its structure.

Due to the considerable amount of non-metal contamination and technical oils in metallic wastes they can't be used as a secondary raw material without preliminary processing. If the question with shavings [1], being pressed in briquettes is partly solved, the existence on the surface of briquetted shavings, oil residues and DRCs leads not only to rapid metal corrosion, but also to the danger of autoignition.

Technologies of processing and enrichment of grinding slimes and wastes of metallurgical productions are so far insufficiently developed for successful launching into production. 
For selection of non-magnetic fraction from powders of magnetic materials, the application of a variation of inhomogeneous magnetic field is considered to be rather perspective.

The magnetic field is created by superposition of the constant and variable strongly inhomogeneous fields. In such a field powders of magnetic materials, under a certain ratio of parameters, form a magneto-vibrating layer in which the destruction of natural aggregates and, as a result, the efficient separation of non-magnetic contaminations takes place.

\subsection{Technology of magneto-vibrating separation of grinding slimes of bearing production}

Assembly of particles, oscillating under the influence of electromagnetic field forces, is considered to view as the area of a magneto-vibrating liquefaction (MVL). Technological processes in the base of which MVL of micronized environment is used become more and more widely spread.

The effectiveness of such technological processes is defined by a number of factors, in particular, by intensity of driving of disperse environment particles [2-4].

To achieve the set up goal it is offered to use separation in the inhomogeneous electromagnetic field that will allow to destroy aggregates, to allocate an abrasive and to get the products of high purity processing [5-7]. Magneto-vibrating separator has to meet the following requirements:

1. The equipment and shipping means of slimes have to be compact for convenient transportation to the next enterprise [8] (for example, owing to exhaustion of stocks of wastes);

2. To process slimes in great amounts, to be ecologically clean and to consume reasonable amount of energy [9];

3. All blocks of the separating device have to be provided with computer-compatible sensors allowing to perform flexible changes of operation parameters of mechanisms and magnetic fields.

A separating device which provides the division of magnetic and non-magnetic fraction of slimes is represented at fig.1.

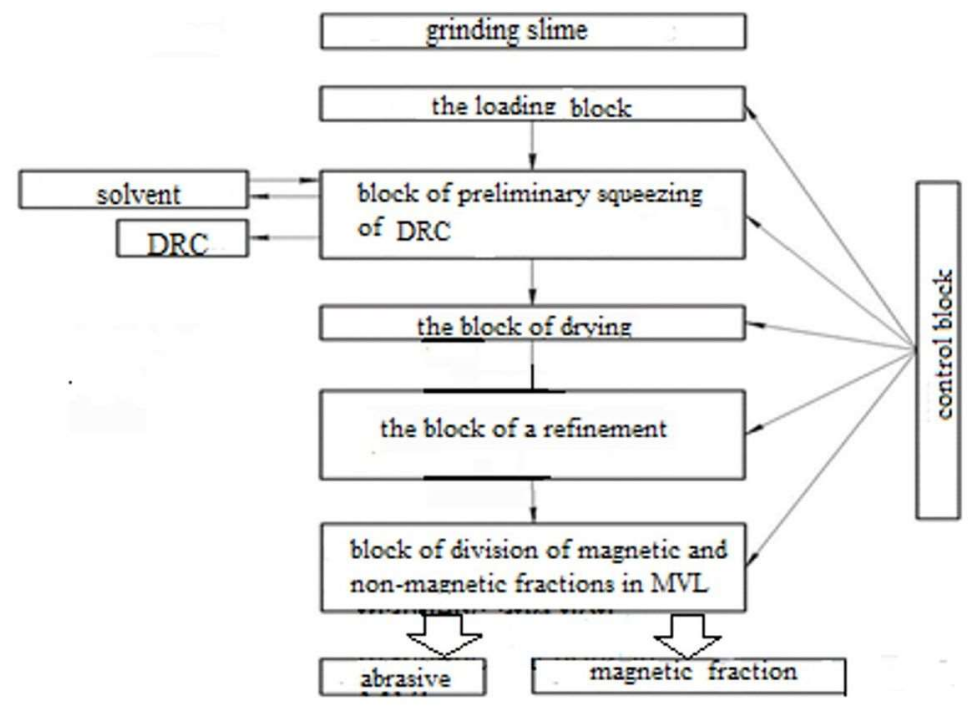


Fig.1. Technological diagram of a separating device.

The complex on processing of grinding slimes includes the following blocks: the loading block with a batcher; block of preliminary squeezing of DRC (centrifuge) and washing of DRC's remains; the block of drying (a conveyor passing through the camera with a temperature $200^{\circ} \mathrm{C}$ ); the block of a refinement (mill); the block of separation of slime fractions.

The block of separation of slime fractions (fig. 2) presents a camera placed between permeameter poles (alternative field electromagnet) and a group of direct field electromagnets [10,11]. Power lines of permeameter fields (2) and direct field magnets (4) are mutually perpendicular. Through the camera (3) the conveyer belt (6) passes on which the separated abrasive settles.

Direct field electromagnets work cyclically for movement of a portion of the pseudoboiling magnetic fraction along the camera to a final mouth (7).

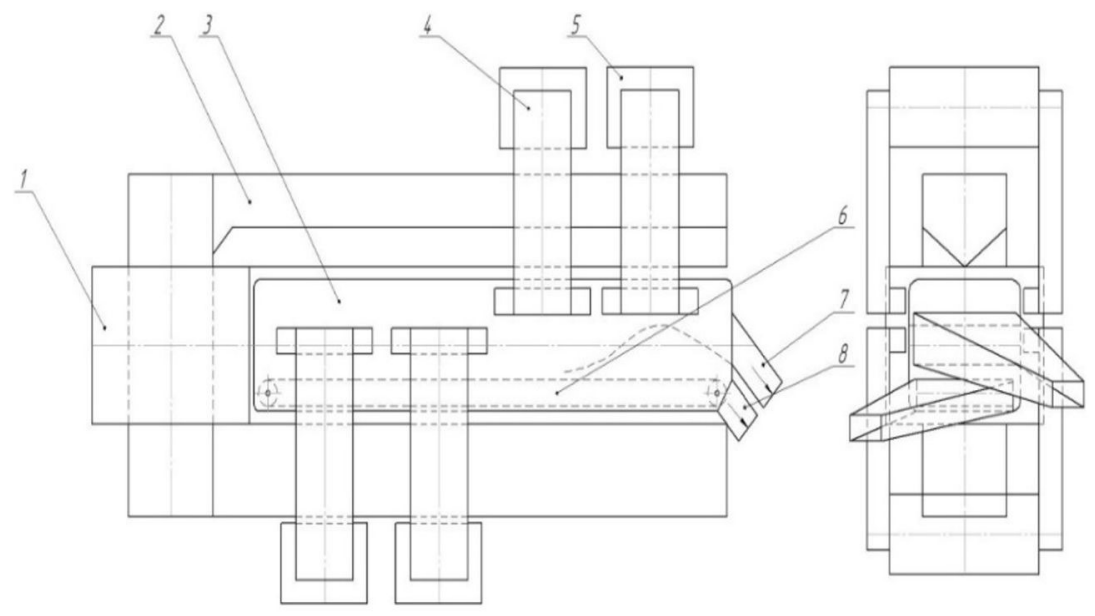

Fig.2 A separating camera of magnetic and non-magnetic slime fractions.

With the entry of slimes in the camera (3), due to the gravity forces of the electromagnetic field of a direct magnet induced in the camera, slimes are being pulled to the area where the magneto-vibrating layer (MVL) is created, therefore there is a process of intensive pseudoboiling of slimes takes place during which an abrasive falls on the bottom of the camera and afterwards is removed by a conveyor belt on the unloading [12-14].

The experimental approbation of the offered technological scheme of slime separation was carried out on an iron ore concentrate of a class of 63 microns with the content of silicon dioxide of $4.57 \%$ of masses [15].

Fig. 3 presents the dependences of silicon dioxide amounts which have fallen out of probe for $120 \mathrm{sec}$. from gradient of the alternative magnetic field induction under various inductions of the direct field. It has been shown that under the field parameters, close to calculated ones, the magneto-vibrating layer of metal powder MP4M has the maximum development. The best results have been reached under $\mathrm{Bc}=12.5 \mathrm{mT}$ and $\mathrm{G}=800 \mathrm{mT} / \mathrm{m}$. Purity of the received metal powder, i.e. effectiveness of the separation process, was determined by a method of the insoluble residue All Union State Standard 16412.8-70 [16]. 


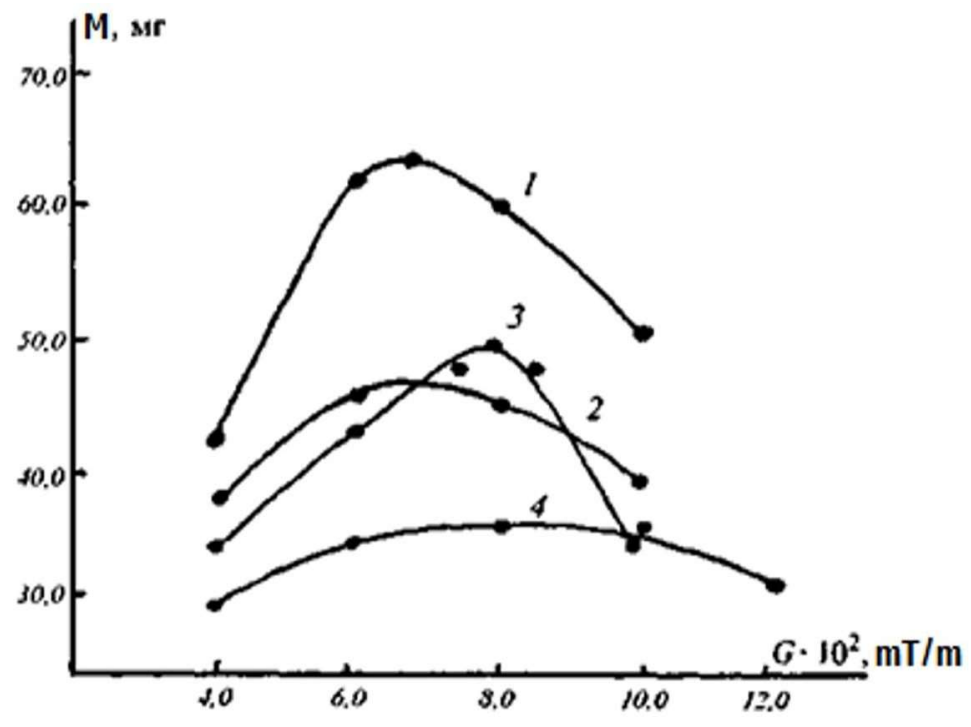

Fig. 3. Dependence of silicon dioxide amounts fallen out of probe for $120 \mathrm{sec}$. from gradient of the alternative magnetic field induction under various inductions of the direct field: $1-B_{c}=7,5 \mathrm{mT} ; 2 \sim$ 10; 3 - 12,5; $4-15 \mathrm{mT}$.

Results of a research on the impact of direct field induction, gradient of an alternative field induction and the time of separation for needed for a process of separation of magnetic and non-magnetic components of grinding slimes of steel SH-15 of GPZ-10 plant are presented at fig. 4 .

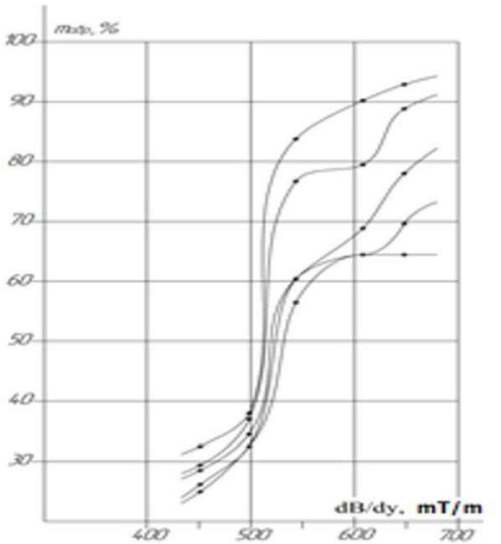

a)

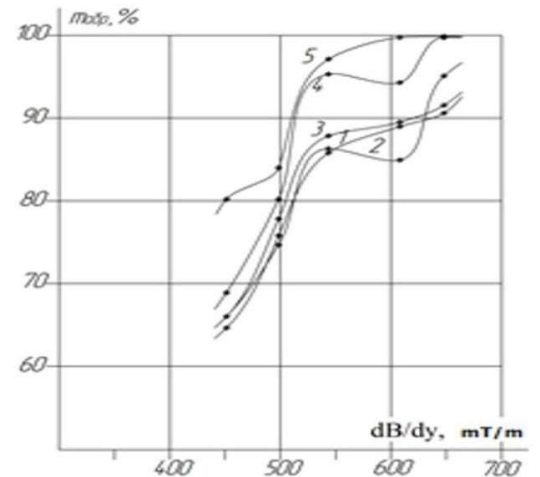

b)

Fig.4. Dependence of the relative mass of the abrasive separated from the gradient of a permeameter field induction $\mathrm{dB} / \mathrm{dy}$ under direct field induction $\mathrm{B}_{\mathrm{c}}=36 \mathrm{mT}$. The time of separation: $\mathrm{a}-150 \mathrm{sec} ., \mathrm{b}-$ $300 \mathrm{sec}$.

On diagrams one can observe the rise in dependence of the mass of separated abrasive on a diagram segment of a gradient of the permeameter field induction from 0.450 up to $0.540 \mathrm{~T} / \mathrm{m}$.

This pattern segment is characterized by weak boiling, but even under the following parameters of magnetic fields, the poorly bound abrasive becomes separated. Further some decrease in mass of the separated abrasive $(0.540-0.610 \mathrm{~T} / \mathrm{m})$ which is explained by the 
unstable mode of a magneto-liquefaction of slimes and the impact of agglomeration process of its particles on separation of an abrasive.

The increase in time of separation leads to more effective separation in the range of a gradient of a field induction from $550 \mathrm{mT} / \mathrm{m}$ up to $610 \mathrm{mT} / \mathrm{m}$.

Fig. 5 presents dependences of quantity of the liberated abrasive at various values of the induction of direct electromagnet field. It has been experimentally proved that under the weak pseudo-boiling of grinding slimes, about $10 \%$ of not separated abrasive remains in metal powder.

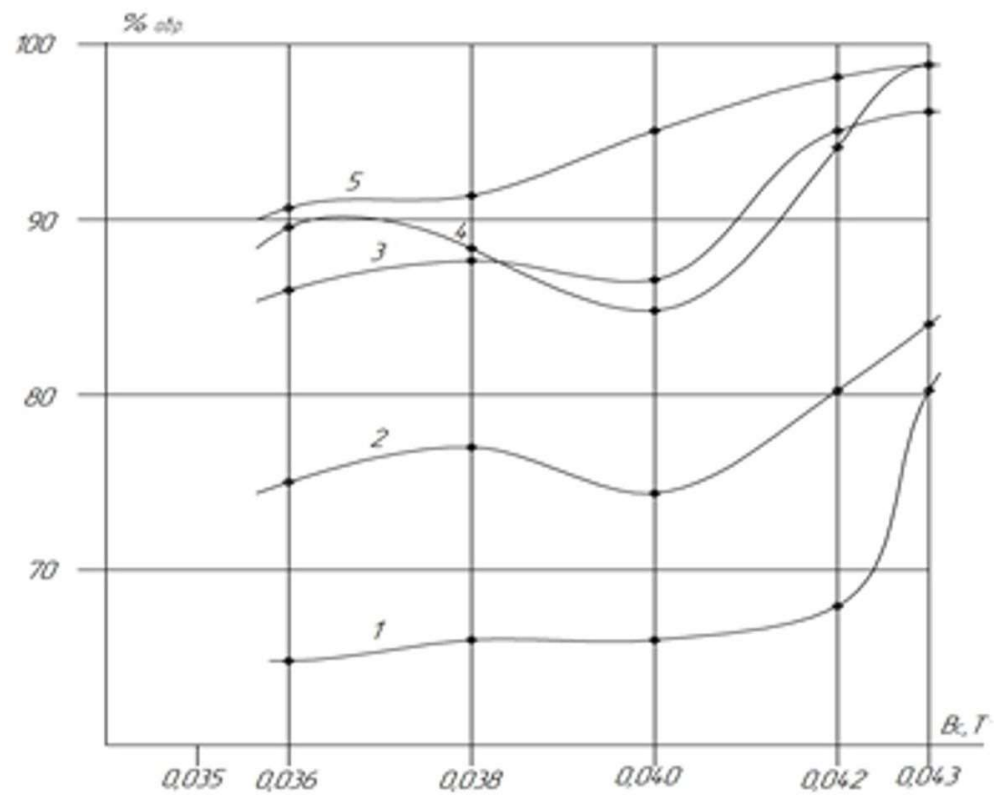

Fig. 5. Dependence of relative mass of the separated abrasive from direct field induction $\mathrm{B}_{\mathrm{c}}$ with the running time of separation $300 \mathrm{sec}$.

$$
\begin{gathered}
\left(1-\frac{d B_{V}}{d y}-0,452 \mathrm{~T} / \mathrm{m}, 2-\frac{d B_{V}}{d y}-0,498 \mathrm{~T} / \mathrm{m}, 3-\frac{d B_{V}}{d y}-0,543 \mathrm{~T} / \mathrm{m},\right. \\
\left.4-\frac{d B_{V}}{d y}-0,609 \mathrm{~T} / \mathrm{m}, 5-\frac{d B_{V}}{d y}-0,648 \mathrm{~T} / \mathrm{m}\right)
\end{gathered}
$$

\section{Conclusion}

The suggested technology of separation of slimes of grinding production in the homogeneous magnetic field, allows to receive high quality processing products, which can be used:

- as raw material for powder metallurgy; additives at the production of filler electrodes;

- for production of pellets for metallurgical production; porous products by methods of condenser welding; productions of monolithic products by methods of high-temperature hydroextrusion; productions of monolithic products with the use of explosion energy;

- in electrometallurgical production;

- in industrial construction materials (production of expanded-clay aggregate);

- as a return for production of the abrasive tool, as well as a non-stick lining coating in foundry production. 
One of the numerous applications of the abrasive restored from grinding slimes is its introduction into foundry production as fire-resistant lining coating of disposable casting moulds on the smelted models.

\section{References}

1. S.S. Kiparisov, O.V. Padalko, Pow. Metal., 9, 56 (1979)

2. M.K. Bologa, S.V. Syutkin, Magn. Hydrodyn., 4, 3 (1981)

3. M.K. Bologa, I.F. Marta, Magn. Hydrodyn., 3, 103 (1988)

4. Y.A. Buevitch, S.V. Syutkin, V.V. Tetyukhin, Magn. Hydrodyn., 4, 3 (1984)

5. V.A. Zlobin, V.A. Andreev, Ferrite materials, (Leningrad, 1970)

6. V.A. Lebedev, A.A. Kochubey, Inter. Scien, and techn, Sym., (Rostov-on-Don, 2015)

7. Y.M. Vernigorov, B.G. Gasanov, S.S. Baev, Sol. S. Phen., 265, 1020 (2017)

8. I.N. Egorov, Y.M. Vernigorov, S.I. Egorova, Euro PM2005 Prague, (Czech Republic,2005)

9. Y.M. Vernigorov, D.M. Plotnikov, V Inter. Conf. on high techn. and basic research (St. Petersburg, 2008)

10. Y.M. Vernigorov, N.N. Frolova, Vest. of Don S. Techn. Un., 11, 7 , (2011)

11. D.W. Haspel, Pelletising waste materials (British Steel Corp, Pat. of England № 2042376)

12. Y.M. Vernigorov, V.A. Lebedev, K.K. Leletko, N.N. Frolova, Inter. Scien. Symp. of mech. Eng. (DSTU, Rostov-on-Don, 2016)

13. Y.M. Vernigorov, K.K. Leletko, Scien. of Eur., 1, 43 (2015)

14. N.N. Belov, N.T. Yugov, D.G. Kopanitsa, Dynamics of high-speed impact and related physical phenomena, (STT, Tomsk, 2005)

15. Y.M. Vernigorov, I.N. Egorov, S.I. Egorova, Vest. of Don S. Techn. Un., 2, 3, (2002)

16. A.A. Kochubey, V.A. Lebedev, Y.M. Vernigorov, Hardening of long parts in a rotating electromagnetic field, (DSTU, Rostov-on-Don, 2018) 